\section{OP0293 A FIRST-IN-CLASS METABOLIC REPROGRAMMING AGENT, MBS2320, SELECTIVELY MODULATES IMMUNE CELL FUNCTION AND IMPROVES OSTEOID FORMATION AND BONE PROTECTION VERSUS ETANERCEPT IN THE MOUSE COLLAGEN-INDUCED ARTHRITIS MODEL}

Lisa Patel ${ }^{1}$, Martyn Foster ${ }^{1}$, Fang Shen ${ }^{2}$, Paul Vink ${ }^{3}$, Ian Anderson ${ }^{2}$, Louise Jopling ${ }^{4}$, Stephen Smith ${ }^{1}$, lain Greig ${ }^{5}$, Sam Williams ${ }^{1}$. ${ }^{1}$ Istesso Ltd, London, United Kingdom; ${ }^{2}$ Johnson and Johnson Pharmaceutical RandD, Spring House, United States of America; ${ }^{3}$ Janssen, Beerse, Belgium; ${ }^{4}$ Johnson and Johnson Innovation, London, United Kingdom; ${ }^{5}$ University of Aberdeen, Aberdeen, United Kingdom

Background: Despite the availability of several treatment options, some Rheumatoid Arthritis (RA) patients do not reach low disease activity. There thus remains a need for differentiated new therapies. Prior studies identified a series of biphenylsulphonamides with bone protecting and anti-inflammatory activity ${ }^{1,2}$ which might meet this need.

Objectives: To evaluate the selectivity of a novel small molecule, MBS2320, in myeloid and lymphoid cells, characterise its selectivity for osteoclasts versus osteoblasts and characterise its effects on synovitis and osteoprotection compared to an anti-TNF $\alpha$ agent in a therapeutically administered collagen-induced arthritis (CIA) mouse model.

Methods: Viability, proliferation or cytokine production were assessed in human primary monocytes, lymphocytes or a Mixed Lymphocytes Reaction (MLR). Human primary osteoclasts (OCs) were differentiated from CD14+ monocytes with M-CSF and RANK-L. Mature OCs were stained with tartrate-resistant acid phosphatase (TRAP) and quantified by light microscopy. Osteolytic activity was assessed on mineral-coated surfaces. Osteoblasts were derived from mesenchymal stem cells by differentiation in the presence of MBS2320; mineralisation was assessed by Alizarin Red S staining. ClA was induced in DBA $/ 1 \mathrm{~J}$ mice by collagen immunisation. MBS2320 and etanercept were dosed once daily after onset of disease. Arthritic Index (Al) was scored throughout the dosing period after which serial paw sections were assessed for inflammation, synovitis, stromal cavity osteolysis, pannus hyperplasia, osteoid layering and bone resorption foci.

Results: MBS2320 reduced the production of cytokine from monocytes and inhibited T-cell proliferation, and cytokine production in a MLR. MBS2320 also reduced primary OC differentiation and function in vitro to a greater degree than alendronate but showed no effect on the differentiation of primary osteoblasts. MBS2320 $(0.3 \mathrm{mpk} / \mathrm{d})$ and etanercept $(10 \mathrm{mpk} / \mathrm{d})$ inhibited the onset and severity of CIA arthropathy, and synovitis, pannus infiltration and osteolysis, with equivalent efficacy. In addition MBS2320 showed anatomically appropriate osteoid layering with conservation of the tide mark, and the bone marrow showed no cell atypia, with all progenitor classes present although reduced in number and distribution. In contrast, osteoid formation in the etanercept group was multi-focal with an often erratic tide-mark morphology and was localised to areas of reduced osteoclastic resorption and osteolysis with erratic osteocyte distribution.

Conclusion: MBS2320 selectively inhibits myeloid and lymphoid activity/differentiation, whilst sparing mesenchymal cells, in vitro. In murine CIA, MBS2320 treatment led to the formation of anatomically appropriate osteoid layering indicating an ongoing process of osteogenesis conditioned by biomechanics. By contrast osteoid formation due to etanercept was more 'reactive' and secondary to suppression of inflammation. The data suggest that MBS2320 offers equivalent antiinflammatory activity, but a broader spectrum of osteoprotective efficacy in CIA compared to TNF $\alpha$ inhibition and may offer an alternative therapeutic approach to improving bone quality in RA.

\section{REFERENCES:}

[1] Greig IR, et al. Development and Characterization of Biphenylsulfonamides as Novel Inhibitors of Bone Resorption J Med Chem 2006;49:7487-7492

[2] Coste $E$, et al. Identification of small molecule inhibitors of RANKL and TNF signaling as anti-inflammatory and antiresorptive agents in mice Ann Rheum Dis 2015;74:220-6

Disclosure of Interests: Lisa Patel Shareholder of: Shareholder of Istesso Ltd, Employee of: Employee of Istesso, Martyn Foster Shareholder of: AstraZeneca, Consultant for: Istesso, Levicept, Employee of: AstraZeneca, Fang Shen Shareholder of: Johnson \& Johnson, Employee of: Johnson \& Johnson, Paul Vink Employee of: Johnson \& Johnson, lan Anderson Shareholder of: Johnson \& Johnson, Employee of: Janssen Research \& Development, LLC, Louise Jopling Shareholder of: Johnson and Johnson (employee), Employee of: Employee of Janssen (Pharmaceutical arm of Johnson \& Johnson) since May 2008 to present day, Stephen Smith Shareholder of: Istesso Ltd, Consultant for: Istesso Ltd, lain Greig Shareholder of: Shareholder in OsteoRx Ltd, a spin-out company from the University of Aberdeen, which retains a financial interest in MBS2133, Sam Williams Shareholder of: Shareholders of Istesso Ltd, Employee of: Employees of Istesso

DOI: 10.1136/annrheumdis-2019-eular.5897

\section{OP0294 \\ TRANSCRIPTIONAL PROFILING OF RA PATIENTS SYNOVIAL TISSUE REVEALS TARGETS FOR PRECISION MEDICINE}

Arthur Mandelin, Shurui Bian, Salina Dominguez, Eric Ruderman, Carla Cuda, Deborah Winter, Harris Perlman. Northwestern University, Medicine/ Rheumatology, Chicago, United States of America

Background: We were the first in the United States to demonstrate the applicability of performing transcriptional profiling of macrophages cells isolated from ultrasound guided synovial biopsies on RA patients (Mandelin et al, A\&R 2018 ${ }^{1}$ ). While the numbers of macrophages are known to corelate with response to therapy in RA patients, little is known about macrophage heterogeneity or dendritic cell (DC) involvement in disease.

Objectives: Here, we performed single cell RNA seq and bulk population RNA seq on RA synovium and peripheral blood (classical monocytes, non-classical monocytes, and DCs) from RA patients in order to compare their genome-wide transcriptional profile within and across individuals.

Methods: We obtained blood samples and ultrasound-guided minimally invasive synovial biopsy tissue from RA patients with active disease. Using Fluorescence Activated Cell Sorting (FACS), we isolated classical monocytes (MHCII+CD14+ +CD16-), non-classical monocytes (MHCII+CD14+CD16+), and dendritic cells (MHCll+CD1C+) from blood. After processing synovial tissue for single-cell suspension, we isolated CD45+ cells for single cell RNA seq and macrophages (MHCll+CD14+CD11b+CD206+) and dendritic cells (MHCll+CD1c+) by FACS We extracted RNA from these cell populations and prepared libraries for RNAseq. These libraries were sequenced on an Illumina NextSeq 500 and assessed for quality of RNA, sequencing, and gene detection.

Results: For each cell population, we assessed the variability of gene expression across patients. We identified 5 different populations of macrophages that differ in their origin, response to methotrexate, differentiation status, and activation status. Additionally, as expected, the bulk population of DCs were highly variable across individuals, which this is likely due to the heterogeneity of subtypes within this population as shown in the single cell RNA seq for macrophages. In circulating monocytes, we observed varying levels of common cytokines and chemokines, such as TNF and CCL1. We also compared gene expression across cell populations to characterize transcriptional signatures that were distinctive to a given cell population. In addition to the genes previously known to be unique to dendritic cells vs. monocytes/macro phages in health, we also identified potential pathogenic factors that varied in their expression across cell types. Finally, to explore the relationship between circulating and tissue cell populations, we asked whether there were pathways that were turned on in the blood prior to extravasation into the synovium. For example, we identified genes that maintained their expression across monocytes and synovial macrophages in RA patients supporting the differentiation of the former into the latter.

Conclusion: Together, these results provide a survey of myeloid cells in the blood and synovial tissue of RA patients. We aim to understand how these cells vary across patients and what clinical variables and medication status influence their transcriptional profile across individuals. Our long-term goal is to use these studies to better understand the underlying mechanisms of pathogenesis and response to current treatments of RA as well as to identify potential targets for future therapies.

\section{REFERENCE:}

[1] Mandelin, A.M., 2nd, et al. Transcriptional Profiling of Synovial Macrophages Using Minimally Invasive Ultrasound-Guided Synovial Biopsies in Rheumatoid Arthritis. Arthritis \& rheumatology (Hoboken, N.J.) 70, 841854 (2018).

Disclosure of Interests: Arthur Mandelin: None declared, Shurui Bian: None declared, Salina Dominguez: None declared, Eric Ruderman Consultant for: Pfizer Inc., Carla Cuda: None declared, Deborah Winter: None declared, Harris Perlman: None declared

DOI: 10.1136/annrheumdis-2019-eular.8317

\section{OP0295 \\ N-LINKED GLYCANS IN THE VARIABLE DOMAIN OF ACPA-IGG IN THE DEVELOPMENT OF RHEUMATOID ARTHRITIS}

Lise Hafkenscheid ${ }^{1}$, Emma C. de Moel ${ }^{1}$, Irene Smolik² ${ }^{2}$ Xiaobo Meng ${ }^{2}$

Stacy Tanner ${ }^{2}$, Bas C. Jansen ${ }^{3}$, Albert Bondt ${ }^{1,4}$, Manfred Wuhrer ${ }^{4}$,

Thomas Huizinga ${ }^{1}$, Rene Toes ${ }^{1}$, Hani El-Gabalawy ${ }^{2}$, Hans Ulrich Scherer ${ }^{1}$.

${ }^{1}$ Leiden University Medical Center, Department of Rheumatology, Leiden,

Netherlands; ${ }^{2}$ University of Manitoba, Arthritis Centre, Winnipeg, Canada; ${ }^{3}$ Ludger

Ltd., Culham Science Centre, Oxfordshire, United Kingdom; ${ }^{4}$ Leiden University

Medical Center, Center for Proteomics and Metabolomics, Leiden, Netherlands

Background: Anti-citrullinated protein antibodies (ACPA) are disease-specific biomarkers in rheumatoid arthritis (RA). Recently, we described that more than 